\title{
SEA-LABS: A Wireless Sensor Network for Sustained Monitoring of Coral Reefs
}

\author{
Matt Bromage ${ }^{1}$, Katia Obraczka ${ }^{1}$, and Donald Potts ${ }^{2}$ \\ ${ }^{1}$ Department of Computer Engineering \\ ${ }^{2}$ Department of Ecology and Evolutionary Biology \\ University of California, Santa Cruz \\ www.ucsc.edu
}

\begin{abstract}
This paper describes SEA-LABS (Sensor Exploration Apparatus utilizing Low-power Aquatic Broadcasting System), a low-cost, power-efficient Wireless Sensor Network (WSN) for sustained, real-time monitoring of shallow water coral reefs. The system is designed to operate in remote, hard-to-access areas of the world, which limits the ability to perform on-site data retrieval and periodic system maintenance (e.g., battery replacement/recharging). SEA-LABS thus provides a customized solution to shallow-water environmental monitoring addressing the trade-offs between power conservation and the system's functional requirements, namely data sensing and processing as well as real-time, wireless communication. We present SEA-LABS' architecture and its current implementation. Finally, we share our experience deploying SEALABS in the Monterey Bay.
\end{abstract}

Keywords: Wireless Sensor Network (WSN), Remote Environmental Monitoring.

\section{Introduction}

Shallow tropical and warm temperate oceans are the major global sites for formation of calcium carbonates and new limestones. These shallow limestones have an important impact on atmospheric carbon dioxide levels. Projected climate changes appear to threaten the calcification potential of many organisms and may reduce the stability of existing limestones. Over the past decade, accumulating evidence indicates that, although the ocean absorbs carbon dioxide (partially ameliorating atmospheric carbon dioxide rise), ocean buffering has been impacted so severely that sea-water chemistry is changing (higher carbonic acid concentration; lower $\mathrm{pH}$ ) at accelerating rates.

Because coral reefs live mainly at land-sea-air "interfaces", they are especially vulnerable to both atmospheric and oceanic changes. The phenomenon of coral "bleaching" is likely to be one visible sign of such stress. Many corals lay down annual skeletal bands with seasonal differences in density and geochemical composition. It is therefore urgent to assess the impact of physical and chemical changes on coral reefs. 
Currently, scientists rely on remote sensing, often through satellite imaging, to monitor coral reef habitats. However, data acquisition, besides being considerably expensive, is far from having adequate temporal and spatial resolution. For instance, typical satellite surveys provide average temperature of the ocean surface over areas of up to 60 square miles. On the other hand, corals living on a reef may experience temperatures that are 5 to 10 degrees Celsius different from that average. Furthermore, scientists do not have access to the data in realtime. In fact, it can take weeks for data to be available and then considerable processing has to happen before scientists have access to useful information.

SEA-LABS aims at building a low-cost, power-efficient wireless sensor network to perform in-situ monitoring of coral reef habitats in order to provide scientists with continuous, real-time data at adequate temporal and spatial resolution.

\section{System Overview}

SEA-LABS consists of two main functional components: sensing nodes affectionately called Programmable Oceanic Devices (PODs) and data sinks (a.k.a. base stations). Each POD is divided into three subsystems: control and processing, wireless communication, and power management (see Figure 1).

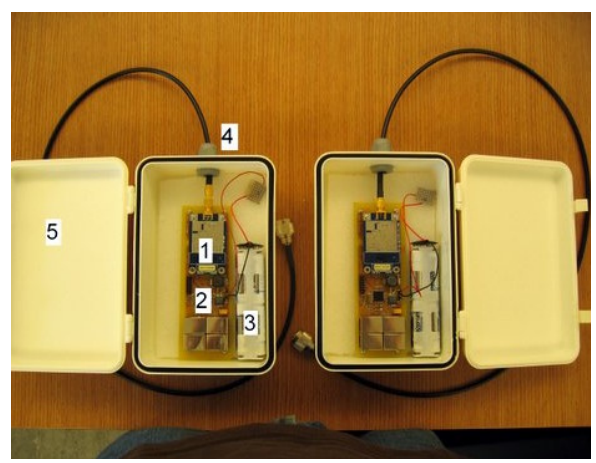

Fig. 1. The POD's hardware, including: (1) the wireless subsystem (2) control and processing subsystem including power management (3) lithium battery pack (4) antenna connector (5) watertight housing

The control and processing subsystem is built around the Texas Instruments MSP430 processor [1, and includes standard sensors (temperature, light, pressure, conductivity, and $\mathrm{pH}$ ).

The wireless subsystem uses the MaxStream 9XTEND 1-Watt transceiver [2] operating in the $900 \mathrm{MHz}$ frequency spectrum. The POD is placed at ocean surface level (e.g., strapped to a buoy) to allow for RF communication.

The power management subsystem implements a custom scheduling algorithm (as opposed to using TinyOS) so that overhead could be minimized. TPS1120 
dual p-channel enhancement mode MOSFETs [3] are used to power down components not being used, thus preserving battery power and increasing the system lifespan.

The base station consists of a laptop connected to the Internet, where the sensor data is ultimately received.

\section{$3 \quad$ Scheduling and Networking}

The scheduler switches the POD through a duty cycle that has four modes of operations, namely: (1) Deep Sleep, (2) Data Collection, (3) Data Analysis, and (4) Wireless Transmission.

During deep sleep, the processor is put into low-power mode, which turns off all unnecessary system clocks and peripherals. To take sensor readings, the POD enters the data collection mode. Afterward, the processor analyzes the results and formats the information into network packets. After successfully sending the data packets, the POD transitions to a deep sleep mode until the next round of sampling.

SEA-LABS' current network implementation follows a star topology with the information sink (or base station) as the middle node and sensing nodes on the edges. The deployment assumes that all sensing nodes are one hop away from the sink. As the basic medium access mechanism, we use a half-duplex slotted-Aloha system 4]. This scheme works well given that the network load requirements are typically low (less than 1 percent). One of the items of future work is to enable multi-hop deployments which will ease current radio coverage requirements.

Once the data packets are received at the base station, it is posted to an online mySQL database. The database can be queried using a PHP graphical user interface (GUI). Users can access information by selecting combinations of sensor readings, POD devices, date and/or time. The user has the option to download the selected data into an Excel spreadsheet for analysis.

\section{Monterey Bay Deployment}

SEA-LABS was deployed in the Monterey Bay as an initial assessment of the systems's functionality and performance in a scenario similar to the target environment. The bay is subject to adverse weather conditions and a non-ideal transmission medium. The deployment's main goals were: (1) validate battery life estimates; (2) test communication capabilities and network functionality; (3) evaluate overall system robustness. A single node was deployed in the Monterey Bay attached to a pre-existing buoy. Initial deployment results are quite promising in that no packets were dropped over the network. Future deployments will employ batteries with increased capacity and new networking protocols to allow multi-hop communication. 


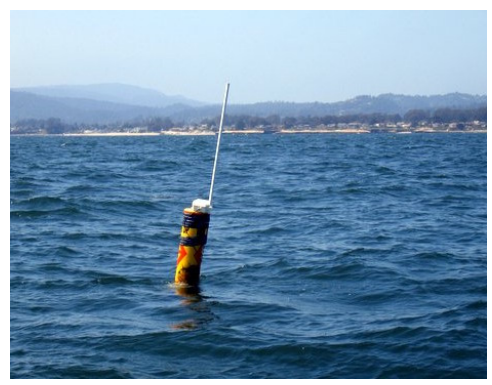

Fig. 2. POD attached to buoy during Monterey Bay deployment

\section{Related Research}

There are several efforts related to SEA-LABS including the Berkeley Mote[5], efforts led by UCLA's Center for Embedded Networked Sensing[6], UC Berkeleys' Wireless and Embedded Systems[7] group, and the ZebraNet [8] project. The Aquaflecks and Amour AUV projects [9] highlight efforts in the area of of underwater autonomous vehicles (AUV).

Acknowledgments. This work has been supported in part by grants from NSF ANI-0322441, CDELSI, the Ferdinand S. Ruth Fund and Myers Trust, the Friends of Long Marine Lab, and Mitubishi Corporation's Global Coral Reef Conservation Project.

\section{References}

1. MSP430x15x and MSP430x16x Data Sheet (SLAS368A), Texas Instruments, March 2003.

2. Engineering Datasheet: MaxStream 9XTEND OEM Module, MaxStream. [Online]. Available: http://www.maxstream.net/products/xtend/oem-rf-module.php

3. Engineering Datasheet: Enhancement Mode Mosfet TPS1120, Texas Instruments. [Online]. Available: http://focus.ti.com/lit/ds/symlink/tps1120.pdf

4. N. Abramson, "The aloha system - another alternative for computer communications," Proceedings of Fall Joint Computer Conference, AFIPS Conference, 1970.

5. A. Woo, "Mote documentation and development information," http://www.eecs. berkeley.edu/〜awoo/smartdust/, 2000.

6. UCLA Center for Embedded Networked Sensing, CENS. [Online]. Available: http:// www.cens.ucla.edu/

7. UCB Wireless and Embedded Systems, WEBS. [Online]. Available: http://webs.cs. berkeley.edu/

8. ZebraNet, ZEBRANET. [Online]. Available: http://www.princeton.edu/ $\mathrm{mrm}^{\mathrm{m}}$ zebranet.html

9. I. Vasilescu, K. Kotay, D. Rus, M. Dunbabin, and P. Corke, "Data collection, storage, and retrieval with an underwater sensor network," Sensys, 2005. 